\title{
Abducens Nerve Paralysis Caused due to Increased Intracranial Pressure: A Case Report and Review of Literatures
}

\author{
Khadijeh Haji Naghi Tehrani ${ }^{1}$, Zahra Morshedian ${ }^{2} \bowtie$
}

Department Neurology, Islamic Azad University, Tehran Medical Sciences Branch, Tehran, Iran

${ }^{2}$ Islamic Azad University, Tehran Medical Sciences Branch, Tehran, Iran

\begin{abstract}
Background: Double vision due to abducens nerve palsy in patients with Pseudotumor cerebri is a very rare finding and usually occurs by increasing in intracranial pressure (ICP) and therefore by the effect of pressure on abducens nerve. Case Report: A 21-year-old woman has referred to our clinic with symptoms of the headaches, double vision along with nausea and vomiting lasting for three months, with no history of the disease, drug consumption, and the only clinical findings was weighing about $20 \mathrm{Kg}$ for a recent year. In examination VI nerve palsy of the left eye, papilledema of both eyes was reported. The computed tomography (CT) and magnetic resonance imaging (MRI) as a diagnostic test for a patient's brain lesions shown normal report. Also, other hormone testing and complete blood count were normal. For the next step patient underwent for lumbar puncture (LP), the patient's cerebrospinal fluid (CSF) pressure was measured more than $120 \mathrm{CmH2O}$. According to the findings of the examination, patient diagnosed with pseudotumor cerebri and underwent for frequent LP, which during that the headaches and double vision symptoms of patient decreased, which indicates that all signs and symptoms of patients caused by pseudotumor cerebri were due to sudden increase in body weight over the past year. Patient prescribed for Acetazolamide and recommended to lose weight with proper diet. For three months of follow-up, symptoms of increased ICP and papilledema have been cleared. Conclusion: The pseudotumor cerebri is manageable by proper diet, and there is no need for bariatric surgery. [GMJ.2017;6(1):66-69]
\end{abstract}

Keywords: Papilledema; Cerebrospinal Fluid; Pseudo-Tumor Cerebri; Lumbar Puncture; Diplopia

\section{Introduction}

Tiopathic intracranial pressure (ICP) inIcrease, which causes a headache, papilledema, tinnitus and visual acuity reduction may lead to abducens nerve paralysis- which is very rare- and cause diplopia [1]. The prevalence is higher in the female within third decades of life and mostly reported in obese patients [2]. The main symptom is a diffuse headache. In most cases, temporarily blurred vision and

\section{GMJ}

o2017 Galen Medical Journal

Tel/Fax: +98 7136474503

PO Box 7193616563

Email:info@gmj.ir transiently impaired vision happened [3]. Increased ICP causes papilledema and visual loss that leads to optic nerve atrophy. Patients Evaluated with computed tomography (CT) or magnetic resonance imaging (MRI) and lumbar puncture (LP) [4]. From causes cerebral venous occlusive disease, meningeal inflammation, endocrine disorders (obesity, Addison's diseases, discontinuation of steroids, high-dose corticosteroids, hypoparathyroidism, hypothyroidism, treatment with

\footnotetext{
Correspondence to:

Khadijeh Haji NaghiTehrani, Department Neurology, Islamic Azad University, Tehran Medical Sciences Branch, Tehran, Iran

Telephone Number: +989121493324

E-mail: dr_tehrani10@yahoo.com
} 
medications, vitamins such as tetracycline, minocycline, nalidixic acid, 13-cis-acid retinoic could be named [5]. To our knowledge, this report is the first case of abducent nerve palsy due to ti idiopathic ICP increase from Iran in the English medical literature.

\section{Case Presentation}

A 21-year-old woman without any history of the disease and intake of medications with a history of weight gaining around $20 \mathrm{~kg}$ in recent year (weight before $68 \mathrm{Kg}$, recent weight $88 \mathrm{Kg}$ ). Patient referred with a chief complaint of constantly pulsating severe diffuse headache till 3 months ago respectively with double vision, nausea, and vomiting. She was referred to an ophthalmologist due to double vision.

Due to papilledema of both eyes, referred to neurology clinic. On physical examination of the patient, papilledema in eyes; left VI nerve palsy and decreased visual acuity in both eyes was evident. Initially, both brain CT scan, MRI with and without contrast to rule out brain space occupying lesions, magnetic resonance venogram to rule out arteriovenous disorders and venous thrombosis performed which were normal. For next step of preliminary tests, coagulation tests were performed for the patient which were normal.

According to the normal coagulation tests and brain imaging, LP was performed and the patient's cerebrospinal fluid (CSF) pressure was measured. The length of $120 \mathrm{cmH} 2 \mathrm{O} \mathrm{CSF}$ of patients with tube leaked out with high pressure.

According to that CSF pressure was reported more than $120 \mathrm{cmH} 2 \mathrm{O}(12-20 \mathrm{CmH} 2 \mathrm{O}=$ normal). The CSF appearance was clear with normal biochemical properties. For the next step electroretinography was done, which B-wave voltage of left eye was less than right eye by the difference of $53 \mathrm{uV}(\mathrm{NL}=10 \mathrm{uV}$, Figure-1). Depending on the hirsutism, a patient referred for consultation with a gynecologist who examined patients for the possibility of polycystic ovarian (PCO).

The ultrasound findings of the ovaries revealed ovarian cysts with thin septa in right dimension. Finally, with regard to the normal hormonal tests and other studies, this possibility was rejected. According to the history of no specific drug and medicine use and no landmarks of hyper-adrenal and hypo-adrenal and hypo-thyroid, also with recent history and current condition, the patient underwent for repeated LP which after each patient symptom of diplopia reduced. Perimetry follow-up of the patient was performed each three months. Eventually, a completely natural visual field in the right eye but symptoms of the mild and moderate disorder in the left eye reported. After the LP prescription of Acetazolamide (500 $\mathrm{mg} / \mathrm{twice}$ a day) was used for completion of treatment that leads to full recovery of a patient with a headache and diplopia.

The patient was advised to lose weight by proper diet, and prescription of acetazolamide was carried out after three months' follow-up of the patient, all signs, and symptoms of patient and papilledema of both eyes were completely healed.

\section{Discussion}

Pseudotumor cerebri is also known as idiopathic intracranial hypertension. Idiopathic means there are no specific and determined causes for this syndrome but on the other hand it has been suggested that these causes act by increase in intracranial venous pressure which followed by [1] elevated venous pressure, which causes impairing in CSF absorption leads to increases in the resistance to outflow of CSF and marked increases in ICP [2].

Some studies determined obesity as a direct cause for intracranial hypertension [1,3]. Pathway of obesity for increasing ICP goes through the increase of intra-abdominal pressure followed by an increase in pleural pressure that by the effect on blood circulatory system increase systemic blood pressure, vascular resistance, renin and aldosterone levels, and finally ICP [4].

This pathophysiology pathway of obesity impedes venous return from the brain, so it causes an increase in intracranial venous pressure and also increases in ICP.

It is parallel and related with pseudotumor cerebri develop $[2,5]$. 

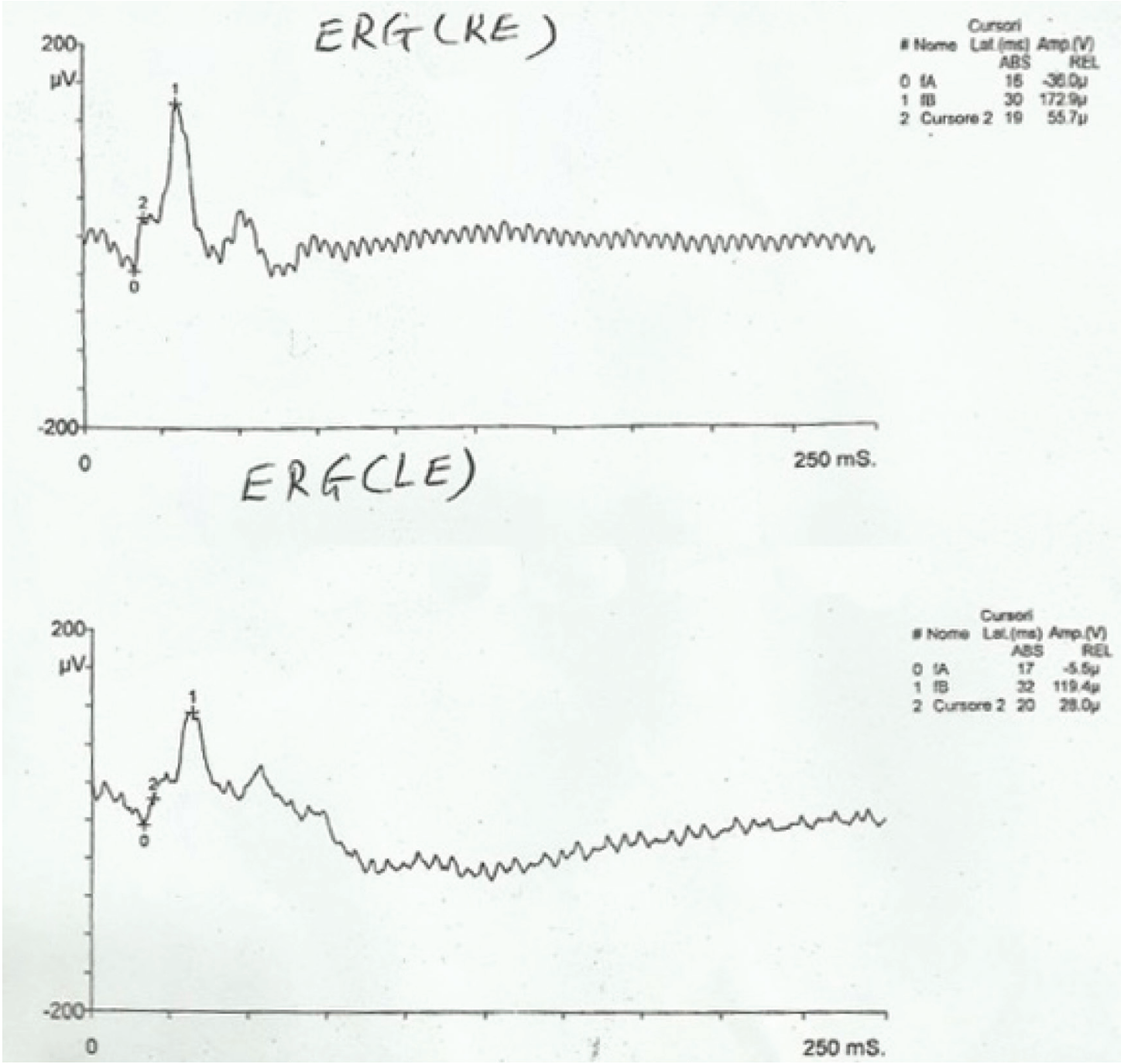

Figure-1. Electroretinography of the patient in the right eye (upper panel) and left eye (lower panel).

In this report, our case shows the role of obesity that causes increasing of ICP through the intracranial venous system. Overweighting in a year caused an increase in abdominal pressure and by its effect caused increasing of ICP which were managed through proper diet and losing weight. Pseudotumor cerebri can obviate by losing weights.

Weight loss has been associated with resolution of marked papilledema [6]. There are not enough evidence that weight loss achieved by diet alone would be as effective as bariatric surgery which causes resolution of pseudotumor cerebri. In this case, the patient obesity and pseudotumor cerebri managed by proper diet and frequent LP. As a result of such order patient's symptoms of high ICP and abducens nerve paralysis completely resolved. This result shows that pseudotumor cerebri is completely manageable by proper diet, and there is no need for bariatric surgery. However, according to evidence hierarchy, this case report is not enough for decision making, and there is a need for further studies.

If weight loss by diet is selected, frequent meetings should manage continuously for the patient which will lead to the success of the program. Further and parallel follow-ups to avoid further loss of vision is critical.

Novel technologies may be useful for prototyping the current intracranial situation of the patient. 
It is an entirely valuable tool for clinicians and medical students to sense patient's status and internal causes lead to an increase in ICP and Abducens nerve paralysis [7]. As the pathophysiology of the effect of obesity on intracranial hypertension was described above, it is best to resolve by losing weights not only by diet but with bariatric surgery in specific cases.

\section{Conclusion}

The pseudotumor cerebri is manageable by proper diet, and there is no need for bariatric surgery.

\section{Conflict of Interest}

The authors state no conflict of interests.

\section{References}

1. Nadkarni T, Rekate HL, Wallace D. Resolution of pseudotumor cerebri after bariatric surgery for related obesity: Case report. J Neurosurg. 2004;101(5):878-80.

2. Karahalios DG, Rekate HL, Khayata MH, Apostolides PJ. Elevated intracranial venous pressure as a universal mechanism in pseudotumor cerebri of varying etiologies. Neurology. 1996;46(1):198-202.

3. Alaud-din A, Meterissian S, Lisbona R, MacLean LD, Forse RA. Assessment of cardiac function in patients who were morbidly obese. Surgery. 1990;108(4):80918; discussion 818-20.

4. Sugerman HJ. Effects of increased intraabdominal pressure in severe obesity. Surg Clin North Am. 2001 Oct;81(5):1063-75.
5. Sugerman HJ, DeMaria EJ, Felton WL 3rd, Nakatsuka M, Sismanis A. Increased intra-abdominal pressure and cardiac filling pressures in obesity-associated pseudotumor cerebri. Neurology. 1997;49(2):507-11.

6. Johnson LN, Krohel GB, Madsen RW, March GA Jr. The role of weight loss and acetazolamide in the treatment of idiopathic intracranial hypertension (pseudotumor cerebri). Ophthalmology. 1998;105(12):23137.

7. Muller A, Krishnan KG, Uhl E, Mast G. The application of rapid prototyping techniques in cranial reconstruction and preoperative planning in neurosurgery. J Craniofac Surg. 2003;14(6):899-914. 